\title{
Development of Tuneable Test Problem Generator for Assembly Sequence Planning and Assembly Line Balancing
}

\author{
Mohd Fadzil Faisae Ab. Rashid ${ }^{1,2}$, Windo Hutabarat ${ }^{1}$ and Ashutosh Tiwari ${ }^{1}$
}

\begin{abstract}
Assembly optimisation activities that involve Assembly Sequence Planning (ASP) and Assembly Line Balancing (ALB) have been extensively studied because of the importance of optimal assembly efficiency to manufacturing competitiveness. Numerous research works in ASP and ALB mainly focuses on developing algorithms to solve problems and to optimise ASP and ALB. However, there is a scarcity in works that focus on developing problems to test these algorithms. In optimisation algorithm development, testing algorithms by a broad range of test problems is crucial to identify their strengths and weaknesses. This paper proposes a generator of ASP and ALB test problems with tuneable complexity levels. Experiments confirm that the selected combination of input attributes does control the generated ASP and ALB problem complexity, and also that the generated problems can be used to identify the suitability of a given algorithm to problem types.
\end{abstract}

\section{Keywords}

Assembly sequence planning, assembly line balancing, test problem generator

\section{Introduction}

In manufacturing, assembly optimisation involves bringing and joining parts and/or subassemblies together to make the process as efficient as possible. Assembly Sequence Planning (ASP) and Assembly Line Balancing (ALB) are classified among major topics in assembly optimisation because both are directly related to assembly efficiency $[1 ; 2]$. Recently, researchers have discovered benefits of solving and optimising ASP and ALB problems together [3; 4], leading to increased research focus on testing new or improved algorithms that operate on these combined problems. In order to assess the performance of new or improved algorithms and to compare them with existing algorithms, a wide range of test problems are required. In ASP and ALB optimisation works that focus on algorithm development or improvement, researchers have used two approaches to test algorithm performance. One approach is to test the algorithms using specific case studies [5; 6]. Another acknowledged approach is to adopt the test problems that are frequently used in literature $[7 ; 8]$. These approaches lack generality because there has been no investigation into the fit of algorithms to problem types. Algorithms have not been tested with a wide range of problem types.

The most frequently used test problem in ASP is an assembly of transmission-type part with eleven components presented by DeFazio and Whitney [9].

\footnotetext{
${ }^{1}$ Manufacturing and Materials Department, Cranfield University, Bedford, UK

2 Faculty of Mechanical Engineering, Universiti

Malaysia Pahang, Pahang, Malaysia

Corresponding author:

Professor Ashutosh Tiwari, Manufacturing and Materials Department, Cranfield University, Bedford, MK43 0AL, United Kingdom. Email: a.tiwari@cranfield.ac.uk
} 
This problem has been presented in many papers such as [10-12] to evaluate algorithm performance. Other than this widely-used problem example, most ASP test problems found in literature have only been used within the same research group. There is thus no accepted standard ASP test problem for evaluating algorithm performance. On the other hand, in ALB optimisation, development of test problems was started in 1960s, resulting in many that have been developed and collected by different researchers. These problems vary in task size from eight to 297 tasks. The famous ALB problems such as the 8-tasks by Bowman, 45 -tasks by Kilbridge and Wester, 70-tasks by Tonge, 111-tasks by Arcus and 297-tasks by Scholl are still being used until today to evaluate algorithm performance for line balancing problems [13].

Although these few benchmark ASP and ALB problems are available for comparing algorithm performance, there is no standard test problem set that covers a wide variety of problem difficulties, especially to test the combined ASP and ALB optimisation. Not only this is important for enhancing the researchers' understanding of their algorithm, it will also help users in selecting which algorithm is more appropriate to their requirements. In order to facilitate such experimentation, a set of problems with controllable complexity level is needed. One way to address this is to devise a test problem generator with tuneable difficulty level that can systematically generate a set of test problems with a desired mix of complexity levels.

This paper proposes a test problem generator with tuneable complexity level for combined ASP and ALB problems. Section 2 explains the requirements and specifications for the proposed test problem generator. Section 3 will explain the methodology of the test problem generator development, which is divided into graph and data generation methodology. Then, section 4 describes the experimental design to test the proposed test problem generator for ASP and ALB. Section 5 presents and discusses the experimental results in this work. Finally, section 6 presents the authors' conclusions on the proposed test problem generator according to experimental results.

\section{Test Problem Generator for ASP and ALB}

In mathematical optimisation community, the importance of test problem generators (TPG) is widely appreciated. Although algorithm development is important, any new algorithm should ideally be tested with a wide range of problem types before making any conclusion on their usefulness [14]. Most of ASP and ALB works focus on proposing and demonstrating algorithm performance on specific ASP and/or ALB problems. There is a lack of investigation into testing and validating the performance of algorithms on wider classes of problems.

A TPG will be useful to provide a wide range of ASP and ALB problems with differing characteristics and difficulties. In many cases, the problem difficulty is only determined by the size of the problem. While this is correct in certain cases, this overlooks the influence of many other attributes on problem difficulty. Additionally, TPG will also be useful to identify which algorithm may be more suitable for a given type of problems. This knowledge is very important to help users to choose the right algorithm, and also for researchers to identify opportunities for further improvement in a particular algorithm. 
To provide the mentioned benefits, the TPG must satisfy the following requirements:

I. Representation. The problems are generated on the basis of assembly task and represented using precedence graph. This is the common way to represent task-based assembly problem in earlier works [2].

II. Output. The TPG is expected to produce precedence graphs that represent taskbased assembly problems. Besides that, the TPG also must be able to generate assembly data, which consists of assembly direction and tool for ASP and assembly time for ALB. These types of data are selected based on popularity from literature survey [2].

III. Tuneable difficulty level. One of the important features expected in a TPG is tuneable difficulty level. This feature will ensure that test problems are generated within known difficulty ranges as required.

There are not many proposals in literature on methods for generating test problems in this domain. Furthermore, existing proposals are limited to generating test problems for ALB. Bhattacharjee and Sahu's proposal is to generate a random precedence graph to represent an ALB problem [15]. In this approach, the assembly problem is generated randomly and then the problem difficulty is measured to determine its complexity level. Later, a systematic data generator for assembly line balancing was proposed by Otto [16]. Besides presenting a systematic method for generating precedence graphs, this work also demonstrates that common graph structures in real-world assembly problems i.e. chains, bottlenecks and modules can be generated on a precedence graph. This approach is also able to generate problems at the desired difficulty levels [16].

Otto's work is the one closest to our stated requirements because this work fulfils the requirements (I) and (III). In Otto's work, ALB problems are generated based on assembly tasks and represented using precedence graphs. It also gives users the ability to create test problems difficulty at the desired level of difficulty. However, since this work was specifically developed for ALB problems, it fails requirement (II). Therefore, in this paper, the ALB-only systematic data generator proposed by Otto in 2011 will be expanded to incorporate both ASP and ALB test problems.

\section{Test Problem Generator Development}

The test problem generator was developed using the methodology presented in Figure 1. The details of each step are explained in section 3.1 to 3.5 .

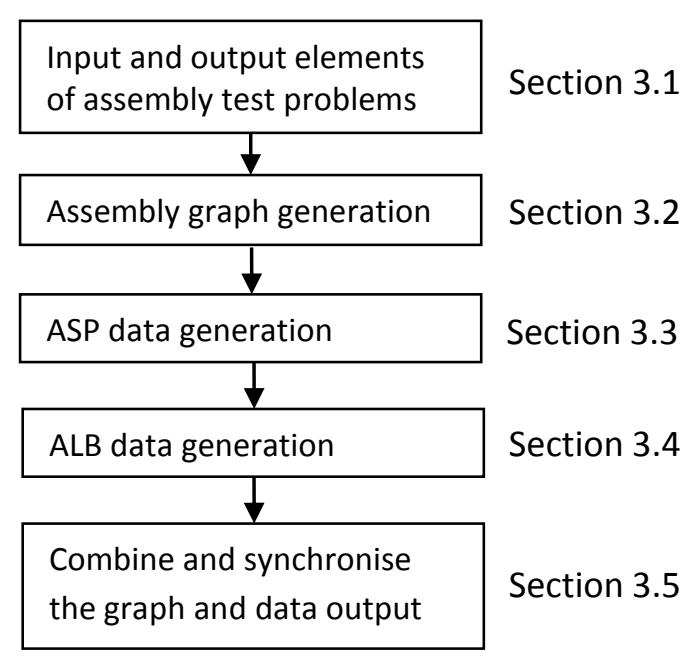

Figure 1: Test Problem Generator Development Flow

The first step in developing the TPG is to identify the input and output elements. Next are the independent development of automated generators for assembly graph, ASP and ALB data. Finally, the outputs from graph and data generators are synchronised and combined to produce a complete test problem set. A worked example of the proposed test problem generator with 
outputs for each step is presented in Table

1.

Table 1: Example of test problem generation process

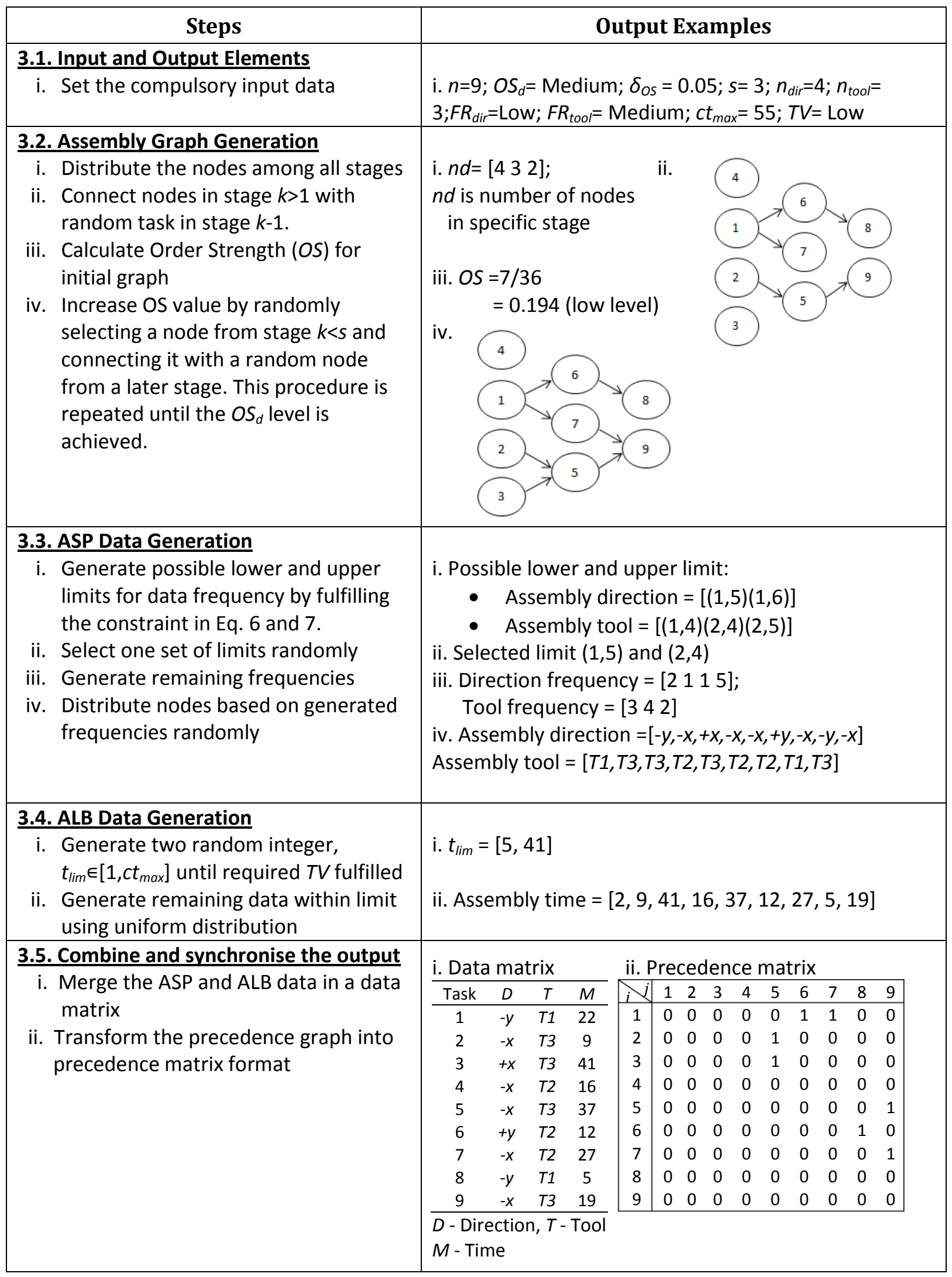




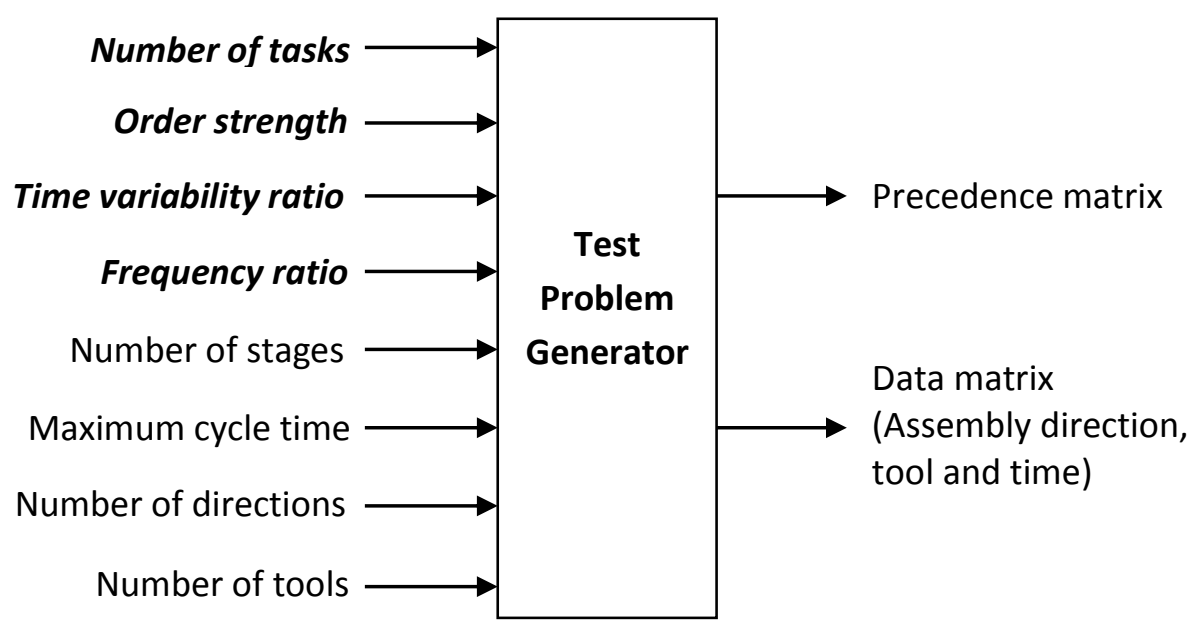

Figure 2: Problem Generator Input and Output Map

\subsection{Input and Output Elements of Assembly Test Problems}

The mapping of input and output variables is shown in Figure 2. The tuneable inputs are presented in bold and italic font.

\subsubsection{Tuneable Input Elements}

The tuneable input elements are variables that are used to control the problem difficulty generated by the TPG. In this work, one new tuning variable is proposed and the rest are adopted from previous works. The TPG is conceptually divided into two parts: the generation of assembly graphs and the generation of assembly data. The next section will discuss the tuneable input variables for each part. Although the tuneable input variables for ALB has been discussed in earlier works, no clear link has been suggested in literature between input and specific difficulty levels for ASP [13].

\section{Tuneable Input for Assembly Graph}

Two tuneable inputs will be used to generate precedence at a specific complexity level. The first input variable to measure graph complexity is $n$, the number of nodes in a graph. In ASP and ALB contexts, graph nodes represent assembly tasks for a given problem. The number of possible assembly sequences will exponentially increase with the number of nodes. In surveyed literature, the size of ASP problems varies between five to 75 nodes; in ALB, $86 \%$ of surveyed ALB papers used between seven to 150 nodes, while the remaining $14 \%$ used up to 300 nodes.

Another graph input variable conceptually linked to graph difficulty is Order Strength. Order Strength $(O S)$ measures the relative number of precedence relation in a graph. By increasing the relative number of precedence relations, the resulting graph is expected to be more complicated [13; 15]. $O S$ is defined as a total number of ordering relation in transitive closure divided by the possible number of ordering relation for particular graph. The $O S$ is calculated as follows.

$O S=\frac{R}{P}$

Eq. 1

$R$ - Total number of ordering relations

$P$ - Possible number of ordering relations

$P=\frac{n(n-1)}{2}$

Eq. 2

$n$ - Number of nodes

The $O S$ value varies between $[0,1] . O S=0$ shows that there is no precedence relation in the graph and $O S=1$ shows that there is 
only one feasible sequence for particular problem. The $O S$ attribute is used together with $O S$ tolerance $\left(\delta_{o s}\right)$ since it is difficult (impossible in some cases) to meet the exact $O S$ value.

\section{Tuneable Input for Assembly Data}

Previously, a number of time-related measures for ALB data have been proposed, such as ratio between maximum and minimum completion time between assembly lines [17], standard deviation [15], and time variability ratio. Time variability ratio (TV) has consistently been used in previous works and is selected for use in this work. $T V$ indicates the range of task time of all tasks dispersed between the assembly lines. TV is calculated as follows:

$$
\begin{array}{ll}
T V=\frac{t_{\text {max }}}{t_{\text {min }}} & \text { Eq. } 3 \\
t_{\text {max }} \geq \frac{1}{3} c t_{\text {max }} & \text { Eq. } 4 \\
t_{\text {max }}-\text { maximum task time } & \\
t_{\text {min }}-\text { minimum task time } & \\
c t_{\text {max }}-\text { maximum cycle time } &
\end{array}
$$

A smaller $T V$ value indicates that existing task times are distributed in a smaller range, which leads to an increased level of problem complexity. The $t_{\max }$ constraint in Eq. 4 is introduced to avoid generation of uniformly small task time, which leads to inconsistency of difficulty levels. The $c t_{\max }$ constraint is explained in section 3.1.2.

Meanwhile, in ASP problem domain, no variable for measuring data complexity has been established. In this work, the ASP data considered are assembly directions and assembly tools. This type of data can be measured by considering how many times (i.e. frequency) a similar direction or tool appears in the problem. A common optimisation objective is to minimise direction or tool changes in a sequence of tasks. Thus, the frequency ratio $(F R)$ is proposed to be used as an input variable that measures ASP data complexity.

$F R=\frac{f_{\min }}{f_{\max }}$

Eq. 5

$f_{\min }-$ Minimum data frequency

$f_{\text {max }}$ - Maximum data frequency

Data with a higher $F R$ is harder to arrange to achieve minimum number of changes because the choice and variability of data are high. This type of data will usually produce higher number of changes compared with smaller $F R$ data. The details of graph and assembly data attributes level are shown in Table 2. In this table, the attribute level for 'number of nodes' is proposed based on a survey on problem sizes as mentioned in section 3.1.1, while the proposed classification of $F R$ and $T V$ levels are based on a few initial tests. The proposed classification of $O S$ levels is adopted from literature review [16].

Table 2: Assembly graph and assembly data attribute levels

\begin{tabular}{llll}
\hline Attributes & Low & Medium & High \\
\hline $\begin{array}{l}\text { Number of } \\
\text { nodes, } n\end{array}$ & $n \leq 20$ & $20<n \leq 70$ & $n>70$ \\
$\begin{array}{l}\text { Order } \\
\text { strength, } O S\end{array}$ & $O S \leq 0.2$ & $0.2<O S \leq 0.6$ & $O S>0.6$ \\
$\begin{array}{l}\text { Time } \\
\text { variability } \\
\text { ratio, } T V\end{array}$ & $T V>6.5$ & $2.5<T V \leq 6.5$ & $T V \leq 2.5$ \\
$\begin{array}{l}\text { Frequency } \\
\text { ratio, } F R\end{array}$ & $F R \leq 0.2$ & $0.2<F R \leq 0.6$ & $F R>0.6$ \\
\hline
\end{tabular}

The tuneable input variables are classified into Low, Medium and High levels because of nonlinearity of the problem. Although the general trend of problem difficulty over tuneable variables can be predicted, when tuning for a targeted difficulty level, too small variable changes may lead to inconsistent difficulty levels. The classification of level difficulties as in Table 2 can be used as a guideline for users in 
selecting appropriate difficulty levels for their use. To reduce the possibility of inconsistent difficulty levels, it is suggested to use the midpoint of the Medium level to generate Medium difficulty problem.

\subsubsection{Other Input Elements}

Apart from the tuneable elements, there are other 'compulsory' inputs that are required for generating a complete problem. Although some of these variables have implications to the problem difficulty level, they are not used here as means to control the problem difficulty because of a lack of agreement in literature. These inputs are: number of stages $(s)$, maximum cycle time $\left(c t_{\text {max }}\right)$, number of assembly direction $\left(n_{\text {dir }}\right)$ and number of assembly tool $\left(n_{\text {tool }}\right)$. Number of stages $(s)$ refers to number of column that contains nodes in a specific precedence graph. In Figure 3, the example graph consists of three stages (hence $s=3$ ) that are shown separated by dotted lines. This variable determines the basic shape of graph, where smaller number of stages will produce graphs with more parallel nodes. The maximum cycle time $\left(c t_{\max }\right)$ is the upper limit of allowable cycle time. This variable is calculated from the required production rate of the assembly line. The number of directions and number of tools are also required to generate ASP data.

Another important element of the TPG is the pseudo-random number generator that underlies most of the data generation algorithm. In this work, the pseudo-random generator used is Mersenne Twister with the range between [0, 232 -1] for 32-bit integer [18]. Appropriate use of seed values ensures that all results are reproducible.

\subsubsection{Output Elements}

There are two sets of outputs generated by the proposed TPG. The first output is the assembly precedence graph (e.g. Figure 3 ), represented by a precedence matrix, which is an $n \times n$ matrix filled with 1 or 0 value $s$ (Table 3). The leftmost column shows assembly tasks and the top row shows the follower tasks. The value 1 shows that the task $j$ must be performed after task $i$.

The second output is a data matrix that consists of assembly directions, assembly tools and assembly time associated with every task. This data is generated according to the required difficulty level as determined in tuneable input variables.

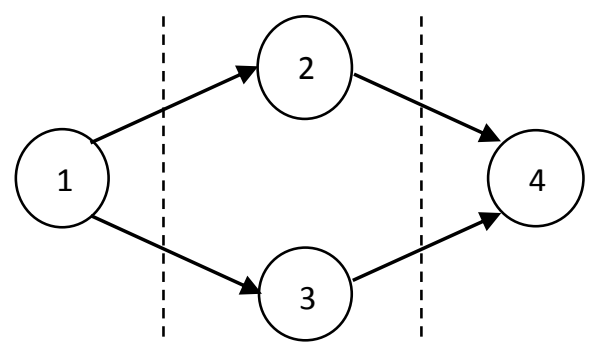

Figure 3: Example of precedence graph

Table 3: Example of precedence matrix

\begin{tabular}{lllll}
\hline \multirow{2}{*}{$i$} & \multicolumn{4}{c}{$j$} \\
\cline { 2 - 5 } & $\mathbf{1}$ & $\mathbf{2}$ & $\mathbf{3}$ & $\mathbf{4}$ \\
\hline $\mathbf{1}$ & 0 & 1 & 1 & 0 \\
$\mathbf{2}$ & 0 & 0 & 0 & 1 \\
$\mathbf{3}$ & 0 & 0 & 0 & 1 \\
$\mathbf{4}$ & 0 & 0 & 0 & 0 \\
\hline
\end{tabular}

\subsection{Assembly Graph Generation}

In this work, the systematic graph generation method is adopted from Otto's work [16]. The five steps below are as proposed in that work.

Step 1: Provide all the compulsory inputs. The compulsory inputs are number of nodes $(n)$, desired Order Strength $\left(O S_{d}\right)$, Order Strength tolerance $\left(\delta_{O S}\right)$ and number of stages $(s)$.

Step 2: Generate and distribute the nodes in all stages using uniform distribution.

Step 3: Connect every node in stage $k>1$ with exactly one random node in stage $k-1$. This step is important to keep the nodes in their original stages. 
Step 4: Calculate the $O S$ using Eq. 1. If the $O S$ is within $O S_{d} \pm \delta_{O S}$, then terminate the process. Otherwise, continue with Step 5.

Step 5: Select a node $i$ in stage $k<s$ and insert an arc to a random node $j$ in stage $m>k$ until the desired $O S$ is achieved. A direct arc from node $i$ to node $j$ is allowed only if:

1. Task $i$ have no restriction such as isolated node or special structure.

2. The $O S$ values have not exceeded the desired upper limit.

\subsection{ASP Data Generation}

In this work, the ASP data that are considered are 'assembly direction' and 'assembly tool change'. The following steps are applied to generate these data. Besides number of tasks, $n$, the required input in ASP data generation is ASP 'data frequency ratio', FR.

Step 1: Calculate all possible lower ( $\left.L_{\text {limit }}\right)$ and upper $\left(U_{\text {limit }}\right)$ limits of data frequencies according to $F R$. The $L_{\text {limit }}$ and $U_{\text {limit }}$ represent the minimum and maximum number of times that a particular direction or tool appear in the generated problem. These limits must fulfil the following constraints:

$U_{\text {limit }} \times n_{\text {type }}-1+L_{\text {limit }} \geq n \quad$ Eq. 6

$L_{\text {limit }} \times n_{\text {type }}-1+U_{\text {limit }} \leq n \quad$ Eq. 7

Eq. 6 and 7 ensure that the summation of generated data within upper and lower limits matches the number of tasks, $n$. In these equations, $n_{\text {type }}$ represent the number of direction $\left(n_{\text {dir }}\right)$ or number of tool $\left(n_{\text {tool }}\right)$ type. In this work, six major direction axes $(+\mathrm{x},-\mathrm{x},+\mathrm{y},-\mathrm{y},+\mathrm{z},-\mathrm{z})$ are considered, thus $n_{\text {type }}$ for $n_{\text {dir }}$ is equal to six. Meanwhile the $n_{\text {type }}$ for $n_{\text {tool }}$ depends on the number of tool types in a particular assembly line.
Step 2: Randomly select a pair of lower and upper limits from the set of possible limits determined in Step 1. Generate remaining data frequencies using uniform distribution. The summation of data frequencies must be equal to $n$.

Step 3: Generate the ASP data based on frequencies (Step 2) in random order.

\subsection{ALB Data Generation}

The ALB data to be generated is the 'task time' for all nodes. The required inputs are 'maximum cycle time' $\left(c t_{\max }\right)$ and 'time variability ratio' (TV). This data is generated in two steps:

Step 1: Calculate all possible limit of task time based on $T V$. The upper limit must not exceed $c t_{\text {max }}$. Randomly select an upper and lower limit from all possible limit pairs.

Step 2: Generate the remaining task times between upper and lower limit using uniform distribution.

\subsection{Combine and Synchronise the Graph and Data Output}

Synchronisation of ASP-specific and ALBspecific outputs is straightforward because both ASP and ALB representations are both developed using the same assembly task basis [19]. Data generated in sections 3.3 and 3.4 are directly linked with assembly tasks and no further adjustment is needed. In this synchronisation step, the output data consisting of ASP data from Step 3.3 and ALB data from Step 3.4 are combined to establish a data matrix. In the data matrix, the assembly direction data is located on the first column, assembly tool data in the second column and assembly data for ALB in the third column.

The final process in this step is to transform the precedence graph into precedence matrix as explained in section 3.1.3. This is 
an important process to synchronise the format of assembly graph into readable computer language.

\section{Experimental Design}

This section describes the setup of the experimental design to assess problems generated using the proposed test problem generator (TPG). The experiment is divided into two phases. In Phase 1, the experiment will focus on the ability of TPG to generate problems at desired complexity level by manipulating the tuneable input attributes. Then, in Phase 2, the generated problems from TPG will be used to evaluate the performance of a set of selected algorithms. The purpose of the second phase experiment is to identify if the generated problems from TPG can be used to characterise the best and worst performance of each algorithm.

\subsection{Phase 1: Testing of Tuneable Input}

The experiment in this phase is conducted by dividing all the tuneable input variables into five levels as presented in Table 4.

Table 4: Tuneable input level setting

\begin{tabular}{ccccc}
\hline Level & $n$ & $O S$ & $T V$ & $F R$ \\
\hline 1 & 15 & 0.2 & 2 & 0.2 \\
2 & 20 & 0.3 & 3 & 0.3 \\
3 & 40 & 0.4 & 4 & 0.4 \\
4 & 60 & 0.5 & 6 & 0.6 \\
5 & 80 & 0.6 & 8 & 0.8 \\
\hline
\end{tabular}

A reference variable setting (datum) is selected as a baseline, while the rest of the problem variable settings are generated by changing only one variable value at a time. In this case, level 3 is selected as the reference variable setting because it is in the middle between minimum and maximum value. The complete experimental table for Phase 1 is shown in Table 5.

From Table 5, 17 test problems are generated by changing one variable at a time. Problem 1 represents the reference variable setting, problem 2 - 5 examine the effect of $n$, problem $6-9$ for effect of $O S$, problem $10-13$ for effect of $T V$ and problem $14-17$ for effect of $F R$.

Table 5: Experimental table for Phase 1

\begin{tabular}{ccccc}
\hline Problem & $n$ & OS & $T V$ & $F R$ \\
\hline $\mathbf{1}$ & $\mathbf{4 0}$ & $\mathbf{0 . 4}$ & $\mathbf{4}$ & $\mathbf{0 . 4}$ \\
2 & 15 & 0.4 & 4 & 0.4 \\
3 & 20 & 0.4 & 4 & 0.4 \\
4 & 60 & 0.4 & 4 & 0.4 \\
5 & 80 & 0.4 & 4 & 0.4 \\
6 & 40 & 0.2 & 4 & 0.4 \\
7 & 40 & 0.3 & 4 & 0.4 \\
8 & 40 & 0.5 & 4 & 0.4 \\
9 & 40 & 0.6 & 4 & 0.4 \\
10 & 40 & 0.4 & 2 & 0.4 \\
11 & 40 & 0.4 & 3 & 0.4 \\
12 & 40 & 0.4 & 6 & 0.4 \\
13 & 40 & 0.4 & 8 & 0.4 \\
14 & 40 & 0.4 & 4 & 0.2 \\
15 & 40 & 0.4 & 4 & 0.3 \\
16 & 40 & 0.4 & 4 & 0.6 \\
17 & 40 & 0.4 & 4 & 0.8 \\
\hline
\end{tabular}

In order to solve precedence graphs, the topological sort algorithm is used to generate feasible assembly sequences. This approach will ensure that the generated sequences are always feasible by sorting the nodes into 'available' and 'unavailable' tasks, during the sequence generation process [20].

To test the generated problems, three different algorithms were selected for each problem type. For ASP problem, a multiobjective genetic algorithm (MOGA) that used in [21] is chosen. This algorithm is selected because, in common with this work, it used task-based representation in 
representing ASP problems. Additionally, genetic algorithm is one of the most frequently used algorithms for solving and optimising ASP problems [2]. In this algorithm, the fitness function for ASP is as follows.

$f_{1}=\frac{d c}{d c_{\max }}+\frac{t c}{t c_{\max }}$ Eq. 8

$d c \quad$ - number of direction changes

tc - number of tool changes

$d c_{\max }$ - maximum possible number of direction changes

$t c_{\max }-$ maximum possible number of tool changes

$d c_{\max }, t c_{\max }-$ number of nodes -1

To test the ALB problem, an ant colony optimisation (ACO) algorithm that has been used for simple assembly line balancing problem (SALBP) in [22] is used. This algorithm is selected based on citation popularity. In addition, ant colony algorithm is also one of frequently used algorithm to solve and optimise ALB problem [2]. In this algorithm, the fitness function is designed as follows.

$f_{2}=\frac{c t}{c t_{\max }}+\frac{n w s}{n w s_{\max }}+\frac{\text { wload }}{w l o a d_{\max }} \quad$ Eq. 9

ct - cycle time

nws - number of workstations

wload - workload variance

$c t_{\max }$ - maximum possible cycle time

$n w s_{\max }$ - maximum possible number of workstations

wload $_{\max }$ - maximum possible workload variance

Finally, for integrated ASP and ALB problem, a Hybrid Genetic Algorithm (HGA) that used in [3] is selected. This algorithm is also selected based on the popularity of this work for integrated ASP and ALB. The fitness function for this problem is designed as follows.

$f_{3}=f_{1}+f_{2}$

$$
\begin{aligned}
& f_{3}=\frac{d c}{d c_{\max }}+\frac{t c}{t c_{\max }}+\frac{c t}{c t_{\max }}+\frac{n w s}{n w s_{\max }}+ \\
& \frac{\text { wload }}{\text { wload }_{\max }}
\end{aligned}
$$

\subsection{Phase 2: Algorithm Testing Using Generated Problems}

In the Phase 2, the algorithms' performance to generate Pareto optimal solution for combined ASP and ALB problem are tested. The purpose of this test to determine whether the problems generated by the TPG have sufficient variety that enables users to perceive differences in algorithm performance. To perform this test, the MOGA and ACO algorithm previously used to optimise ASP and ALB independently will be used to optimise combined ASP and ALB problem alongside Hybrid GA. The objective function set for this experiment is as follows.

$f_{1}=$ minimise number of direction change

$f_{2}=$ minimise number of tool change

$f_{3}=$ minimise cycle time

$f_{4}=$ minimise number of workstation

$f_{5}=$ minimise workload deviation

In order to evaluate the performance of each algorithm when dealing with different complexity problems, the following performance indicators adopted from [23] and [24] are used.

i. Number of nondominated solution in Pareto optimal, $\tilde{\eta}$ : Show the number of nondominated solution generated by each algorithm in Pareto solution. Higher $\tilde{\eta}$ indicates better algorithm performance.

ii. Error Ratio, ER: ER is given by dividing the number of solutions which are not members of the Pareto optimal set with the total number of solutions generated by algorithm q. Smaller ER indicates better algorithm performance. 
iii. Generational Distance, $G D$ : $G D$ finds an average distance of solution with the nearest Pareto optimal solution. Smaller GD indicates better algorithm performance.

$G D_{q}=\frac{s_{i=1}^{s} d_{i}}{s_{q}}$

Eq. 11

$s_{q}$ - number of solutions generated by algorithm $q$

$d_{i}=\min _{k=1}^{P} \overline{{ }_{m=1}^{M}\left(f_{m}^{(i)}-f_{m}^{*(k)}\right)^{2}}$

Eq. 12

Where $f_{m}(i)$ is the $m$-th objective function value of solution $i$ and $f_{m}{ }^{*}(k)$ is the $m$-th objective function value of $k^{\text {th }}$ member of Pareto optimal set.

iv. Spacing: This indicator measures the relative distance between each solution.

Spacing $=\overline{\frac{1}{N}{ }_{i=1}^{s_{q}}\left(d_{i}-d\right)^{2}}$

Eq. 13

$d_{i}$ is distance between solution $i$ and the nearest solution, while $d$ is average of all $d_{i}$. Smaller Spacing indicate better uniformity of space between solutions.

v. Maximum Spread, Max $x_{\text {spread: }}$ Measures the extent of solution distribution found by the algorithm. Larger maximum spread is better.

Max $_{\text {spread }}=\overline{M_{i=1}^{M}\left(\min f_{i}-\max f_{i}\right)^{2}}$

Eq. 14

\section{Results and Discussion}

\subsection{Phase 1 Results}

The output from Phase 1 experiments are presented in Figure 4 to Figure 7, showing the average of best fitness value from ten runs.

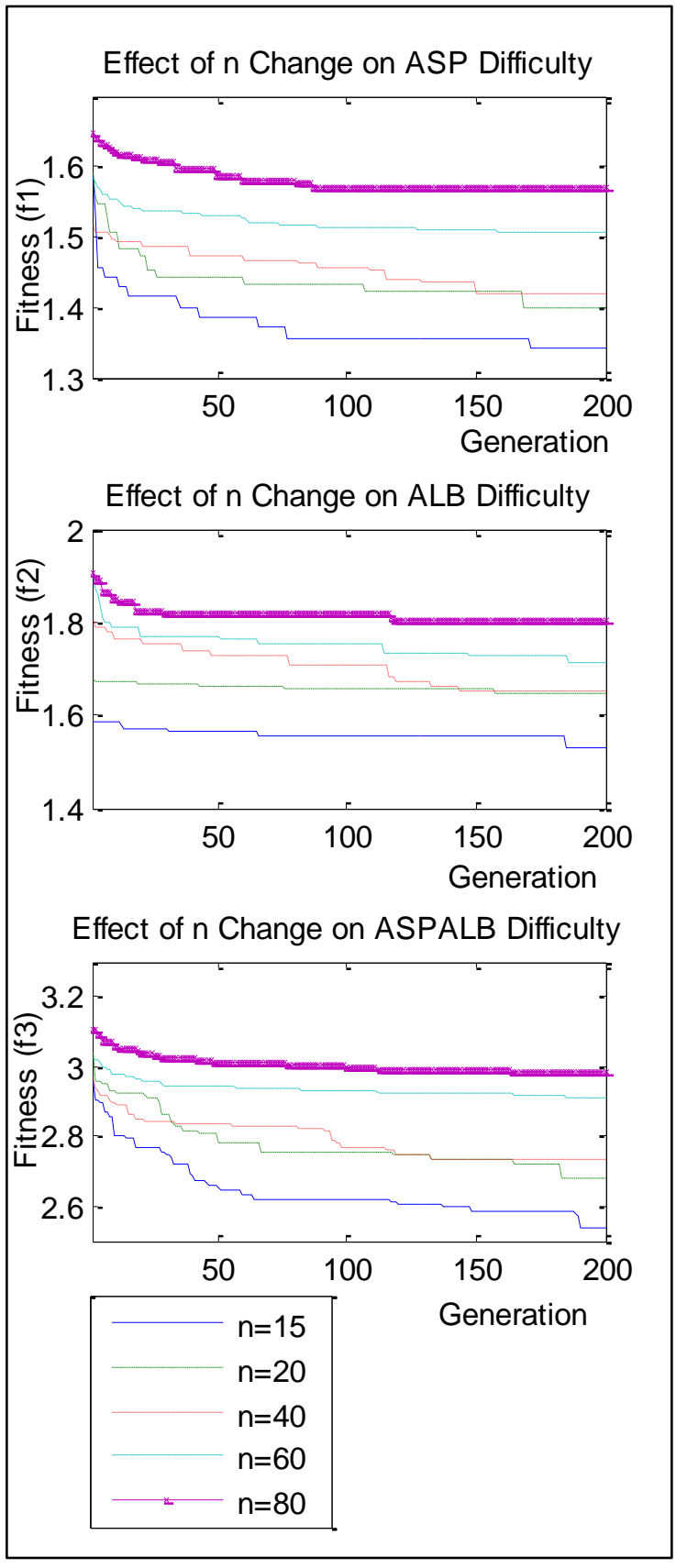

Figure 4: Average of best fitness for a range of $n$ (number of tasks)

Number of tasks ( $n$ ) Figure 4 shows the effect of $n$ on the ASP, ALB and combined ASPALB problem difficulties. In all cases, the problems with larger number of task tend to be found to have better fitness although they have similar tuneable input setting for $O S, F R$ and $T V$. This output pattern is related with increment of problem difficulties when the number of tasks is increased. The output trend is also consistent with previous works such as in 
Scholl (2003), Bhattacharjee (1990) and Otto $(2011)[13 ; 15-16]$.

Order Strength (OS) Figure 5 show the effect of $O S$ change for ASP problems with $15,20,40,60$ and 80 tasks. In these graphs, the ASP problems with high $O S$ values tend to produce better fitness values compared with low and medium $O S$ values. A similar output pattern is also found in ALB and combined ASPALB problems as shown in Figure 5. This result indicates that problems with higher $O S$ values will have lesser difficulty levels compared with low $O S$ values. This finding corroborates a few previous works [25-27], while contradicting a few works that associate higher $O S$ values with greater complexity $[13 ; 16]$.

This mismatch is due to the dissimilar approaches used in solving the precedence graph. In the works that directly used generated permutation as assembly sequence, precedence graphs with higher OS values are harder to solve. Direct permutation has high probability of generating infeasible sequences; since the numbers of precedence constraints in high $O S$ graphs are higher than low $O S$ graph while the search space for both conditions remains the same.

On the other hand, in the works that ensures the feasibility of sequence such as using topological sort, the precedence graph with higher $O S$ is easier to solve, because of differences in search space size. The $O S$ value directly influences the number of possible feasible sequence in a precedence graph. In this case, the number of feasible sequences in high $O S$ is smaller than in low OS because the precedence constraints limit the flexibility of re-sequencing. Since the search space for the precedence graph with high $O S$ is smaller than low $O S$, it is easier to generate solution with better fitness in high $O S$ graphs than with low $O S$ graphs.

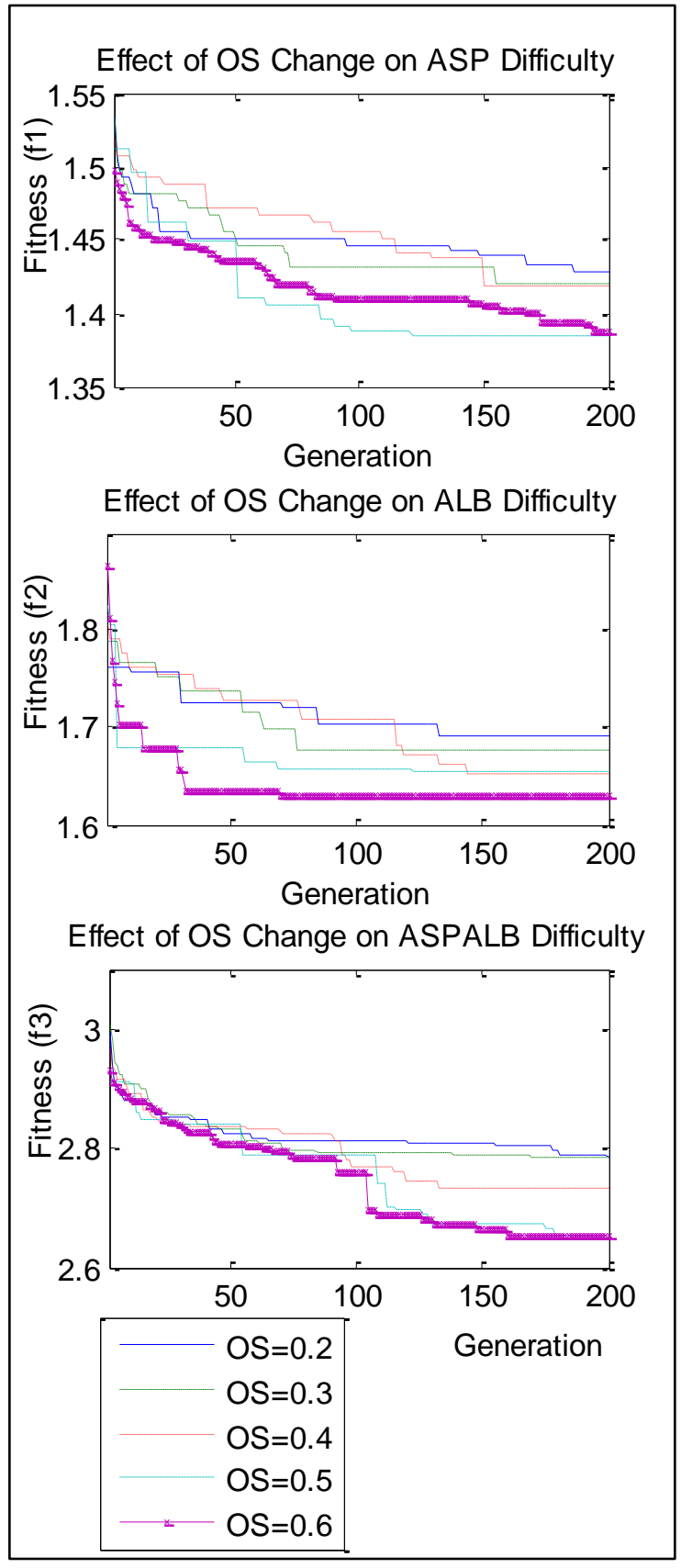

Figure 5: Average of best fitness for different $O S$ value

Nevertheless, there is inconsistency in outputs for ASP with OS 0.5 and 0.6, ALB with $O S 0.4$ and 0.5 and combined ASPALB with $O S 0.5$ and 0.6. For these cases, the problem with smaller $O S$ emerges with better fitness compared with larger OS. A likely explanation is that the chosen OS gaps for these problems are too small, since it does not happen in larger $O S$ gaps such as between $O S 0.6$ and 0.4 or smaller. Small $O S$ gap means that there is only small search 
space difference between the two problems that has influenced the inconsistency of results for both conditions. Therefore, to ensure a clear separation between one difficulty levels with another, $O S$ gaps which are too small should be avoided. More investigation is needed to fully investigate the effect of OS.

Frequency Ratio (FR) The output from ASP problem in Figure 6 shows that the proposed complexity attributes $F R$ can be used to control the ASP data complexity.

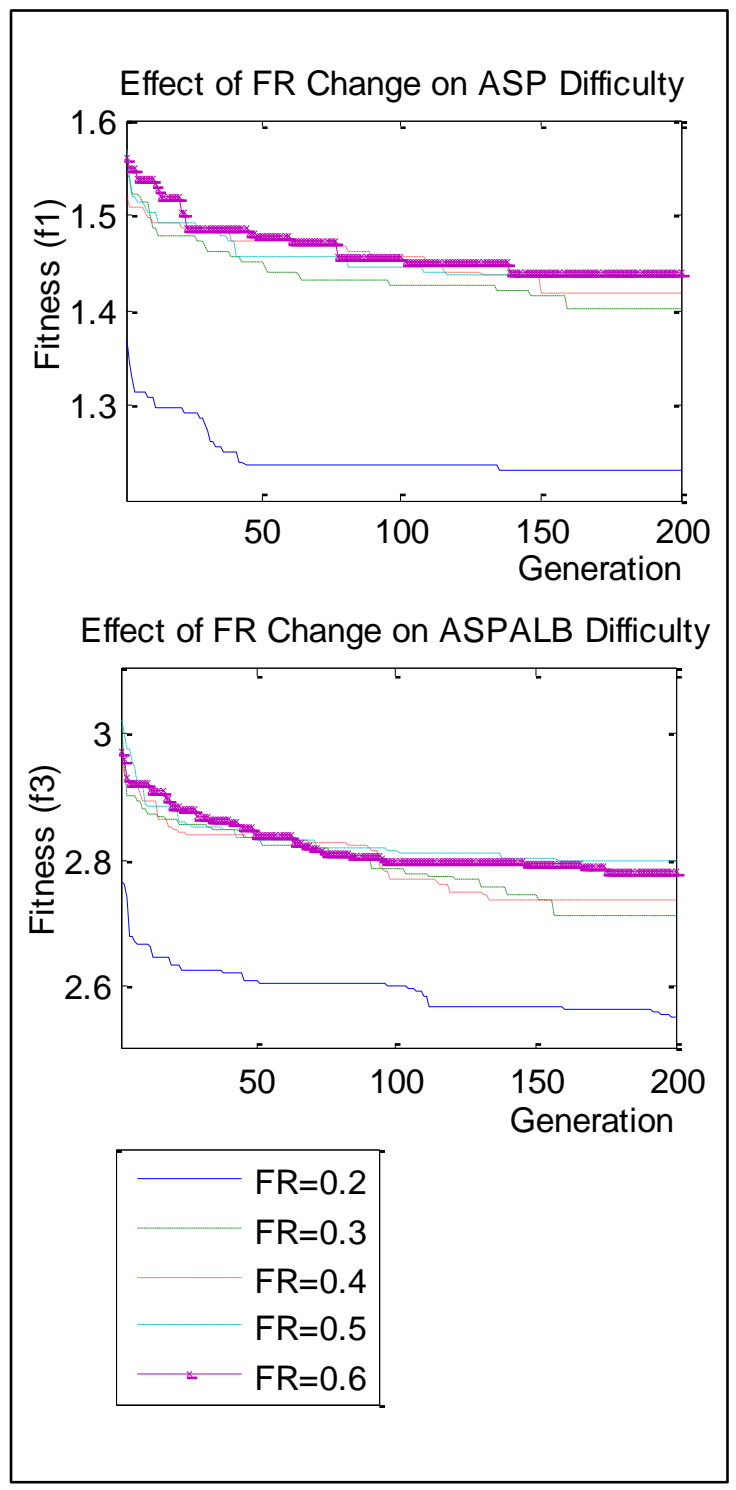

Figure 6: Average of best fitness for different Frequency Ratio
ASP data with high $F R$ will have wider range of choices that directly increase the size of search space. In contrast, ASP data with low $F R$ have smaller search space due to a more limited data variety. As a consequence, the algorithms found it more difficult to achieve minimum direction and tool change for ASP data with higher $F R$.

Time Variability ratio (TV) ALB results in Figure 7 confirm that the Time Variability ratio (TV) adopted from previous works is effective to control the assembly time data complexity $[13 ; 16]$.

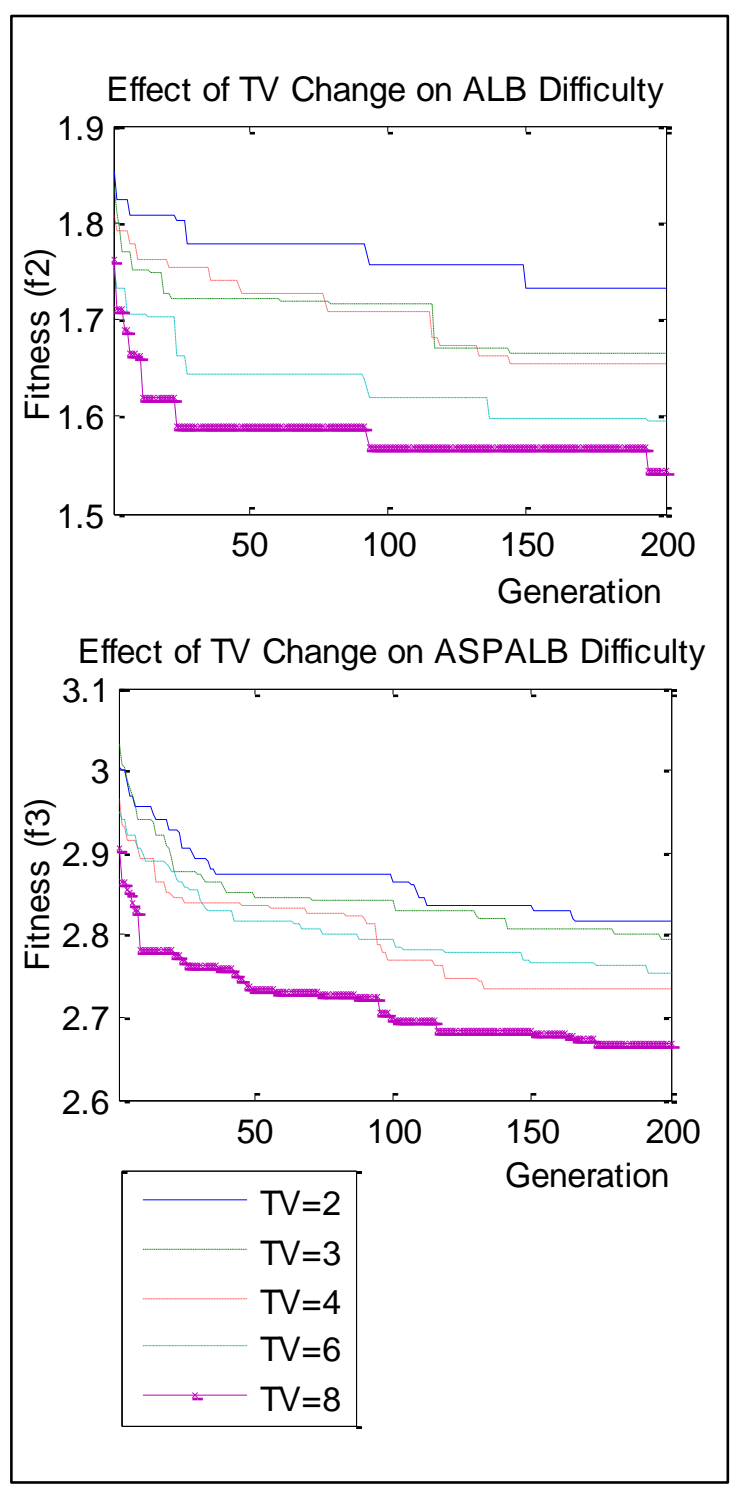

Figure 7: Average of best fitness for different Time Variability Ratio 
The assembly times with higher $T V$ are easier to arrange because the combination of small and large task times tend to fit the cycle time better than uniformly large task times (low TV). Finally the combined ASPALB outputs in this figure clearly show that the TV input variable is able to control the assembly data difficulties as expected.

The results of tuneable input test show that ASP and ALB problem complexity can be controlled via the input attributes of the test problem generator. Although the early assumption that the precedence graph with higher $O S$ will have greater complexity is unfounded, this attribute's usefulness is maintained by redefining its value: to generate precedence graphs with low complexity, higher $O S$ level must be used, while for graphs with high complexity, the OS must be set to the lower level. It is found that the selection of tuneable input level is also important to ensure that the desired problem difficulty is achieved. Selection of proper gaps between one level to another is very important to avoid inconsistent problem difficulty.

In order to test the significance of the results, statistical tests are performed. In this case, ANOVA test is carried out to test if there are any significant differences between the results of one level with results from another level. The null hypothesis stated that there would be no difference among five tuneable input levels means. The summary of ANOVA test is presented in Table 6.

In this case, the critical $f$-value $\left(f^{*}\right)$ that is acquired with 0.05 level of significance from $f$-distribution table is 2.22 [28]. Table 6 consistently shows larger $f$-values compared with $f^{*}$. Since all the $f$-values are larger than $f^{*}$, the null hypothesis for all tuneable input are rejected. In other words, it shows that at 0.05 confidence levels, there are statistically significant differences between levels for $n, O S, F R$ and $T V$.

Table 6: Summary of ANOVA test

\begin{tabular}{|c|c|c|c|c|}
\hline & $\begin{array}{c}\mathrm{n} \\
\text { Change }\end{array}$ & $\begin{array}{c}\text { OS } \\
\text { Change }\end{array}$ & $\begin{array}{c}\text { FR } \\
\text { Change }\end{array}$ & $\begin{array}{c}\text { TV } \\
\text { Change }\end{array}$ \\
\hline SSB & 16.4897 & 0.9418 & 7.3437 & 2.5119 \\
\hline SSW & 3.2268 & 3.7542 & 2.181 & 2.1205 \\
\hline SST & 19.7165 & 4.6961 & 9.5247 & 4.6324 \\
\hline MSB & 4.1224 & 0.2354 & 1.8359 & 0.6280 \\
\hline MSW & 0.0032 & 0.0037 & 0.0021 & 0.0021 \\
\hline$f$ & 1288.25 & 63.62 & 874.23 & 299.05 \\
\hline SSB - Sum of square between groups \\
SSW - Sum of square within groups \\
SS - Sum of square total \\
MSB - Mean squares between groups \\
MSW - Mean squares within groups
\end{tabular}

However, this test does not tell us the exact groups or levels that have statistically significant difference in means. Therefore, an a posteriori test known as Tukey's Honestly Significant Different test (Tukey's HSD test) is performed.

Tukey's HSD test compares the mean of rejected null hypothesis with the means of other groups to identify if there is any significant difference between the mean of one level with another. The value of the absolute difference between two means will be compared to a critical HSD as proposed in the Tukey's table [28]. The summary of Tukey's HSD test at 0.05 confidence interval is presented in Table 7.

From Table 7, the absolute mean difference between two levels for $n$ and $T V$ consistently indicates larger values than the critical HSD value. It shows that there are significant difference in all levels for $n$ and $T V$. It means that the problem difficulties for these variables can be statistically distinguished between each level. 
Table 7: Summary of Tukey's HSD test

\begin{tabular}{|c|c|c|c|}
\hline $\begin{array}{c}\text { Variable } \\
\text { (critical HSD) }\end{array}$ & \multicolumn{2}{|c|}{$\begin{array}{c}\text { Comparison } \\
\text { Level }\end{array}$} & \multirow{2}{*}{$\begin{array}{c}\begin{array}{c}\text { Absolute } \\
\text { Mean } \\
\text { Difference }\end{array} \\
0.1331\end{array}$} \\
\hline \multirow{10}{*}{$\begin{array}{c}n \\
(0.015536)\end{array}$} & 15 & 20 & \\
\hline & 15 & 40 & 0.1497 \\
\hline & 15 & 60 & 0.2929 \\
\hline & 15 & 80 & 0.3658 \\
\hline & 20 & 40 & 0.0166 \\
\hline & 20 & 60 & 0.1598 \\
\hline & 20 & 80 & 0.2327 \\
\hline & 40 & 60 & 0.1432 \\
\hline & 40 & 80 & 0.2161 \\
\hline & 60 & 80 & 0.0729 \\
\hline \multirow{10}{*}{$\begin{array}{c}O S \\
(0.016759)\end{array}$} & 0.2 & 0.3 & 0.008 \\
\hline & 0.2 & 0.4 & 0.0292 \\
\hline & 0.2 & 0.5 & 0.068 \\
\hline & 0.2 & 0.6 & 0.0755 \\
\hline & 0.3 & 0.4 & 0.0212 \\
\hline & 0.3 & 0.5 & 0.06 \\
\hline & 0.3 & 0.6 & 0.0675 \\
\hline & 0.4 & 0.5 & 0.0388 \\
\hline & 0.4 & 0.6 & 0.0169 \\
\hline & 0.5 & 0.6 & 0.0075 \\
\hline \multirow{10}{*}{$\begin{array}{c}F R \\
(0.012773)\end{array}$} & 0.2 & 0.3 & 0.192 \\
\hline & 0.2 & 0.4 & 0.1954 \\
\hline & 0.2 & 0.5 & 0.2301 \\
\hline & 0.2 & 0.6 & 0.2256 \\
\hline & 0.3 & 0.4 & 0.0034 \\
\hline & 0.3 & 0.5 & 0.0381 \\
\hline & 0.3 & 0.6 & 0.0336 \\
\hline & 0.4 & 0.5 & 0.0347 \\
\hline & 0.4 & 0.6 & 0.0302 \\
\hline & 0.5 & 0.6 & 0.0045 \\
\hline \multirow{10}{*}{$\begin{array}{c}T V \\
(0.009053)\end{array}$} & 2 & 3 & 0.0205 \\
\hline & 2 & 4 & 0.0708 \\
\hline & 2 & 6 & 0.0894 \\
\hline & 2 & 8 & 0.1453 \\
\hline & 3 & 4 & 0.0503 \\
\hline & 3 & 6 & 0.0389 \\
\hline & 3 & 8 & 0.1248 \\
\hline & 4 & 6 & 0.0114 \\
\hline & 4 & 8 & 0.0745 \\
\hline & 6 & 8 & 0.0859 \\
\hline
\end{tabular}

Meanwhile, in $O S$ and $F R$ variables, the absolute mean difference also shows larger values than critical HSD except for the cases between $O S$ values of 0.2 and $0.3, O S$ values 0.5 and $0.6, F R$ values 0.3 and 0.4 , and $F R$ values 0.5 and 0.6 . This result is related with selection of appropriate gaps between levels, since it only occurs between adjacent levels. Consistent with earlier discussion on the effect of $O S$ change on the problem difficulties (Figure 5), too small gaps between consecutive levels should be avoided.

\subsection{Phase 2 Results}

In this phase, 25 problems with different difficulty settings are used to demonstrate the usefulness of the TPG for testing the performance of algorithms. The setup is for multi objective optimisation of ASP, ALB, and ASPALB problems. The assembly problem for this experiment is set up as in Table 8 and Table 9.

Table 8: Problem setting for experiments in Phase 2

\begin{tabular}{|c|c|c|}
\hline Problem & $\begin{array}{c}\text { Graph } \\
\text { Difficulties }\end{array}$ & $\begin{array}{c}\text { Data } \\
\text { Variables }\end{array}$ \\
\hline 1 & Low & Low \\
2 & Low & Low-Med \\
3 & Low & Medium \\
4 & Low & Med-High \\
5 & Low & High \\
\hline 6 & Low-Med & Low \\
7 & Low-Med & Low-Med \\
8 & Low-Med & Medium \\
9 & Low-Med & Med-High \\
10 & Low-Med & High \\
\hline 11 & Medium & Low \\
12 & Medium & Low-Med \\
13 & Medium & Medium \\
14 & Medium & Med-High \\
15 & Medium & High \\
\hline 16 & Med-High & Low \\
17 & Med-High & Low-Med \\
18 & Med-High & Medium \\
19 & Med-High & Med-High \\
20 & Med-High & High \\
\hline 21 & High & Low \\
22 & High & Low-Med \\
23 & High & Medium \\
24 & High & Med-High \\
25 & High & High \\
\hline & & \\
\hline
\end{tabular}


Table 9: Attribute settings for different graph and data difficulty levels

\begin{tabular}{|l|c|c|c|c|}
\hline \multirow{2}{*}{ Level } & \multicolumn{2}{|c|}{$\begin{array}{c}\text { Graph } \\
\text { Difficulty }\end{array}$} & \multicolumn{2}{c|}{$\begin{array}{c}\text { Data } \\
\text { Difficulty }\end{array}$} \\
\cline { 2 - 5 } & $n$ & $O S$ & $F R$ & $T V$ \\
\hline Low & 15 & 0.6 & 0.2 & 8 \\
\hline Low-Med & 20 & 0.5 & 0.3 & 6 \\
\hline Med & 40 & 0.4 & 0.4 & 4 \\
\hline Med-High & 60 & 0.3 & 0.5 & 3 \\
\hline High & 80 & 0.2 & 0.6 & 2 \\
\hline
\end{tabular}

The tuneable input for these test problems are grouped into Graph Difficulty $(n$ and $O S$ variables) and Data Difficulty (FR and TV variables). The data of test problems that are used in this work can be downloaded from the following website: http://public.cranfield.ac.uk/s135489/ASP_ ALB_Test_Problems_Data.zip.

The results from Phase 2 experiments are summarised in Table 10. Numbers in brackets are weighting values that are assigned to each algorithm based on its performance for the respective indicator. For every indicator in a given problem, the best result is assigned weight value 3 , while the second and third positions are assigned weight values 2 and 1 respectively. Then, the algorithm ranking is made through comparison of the weighted sums.

Table 10: Summary of the result of experiments on selected multi-objective algorithms

*Numbers in brackets are weighting values from the best (weight=3) to worst (weight=1) performance.

\begin{tabular}{|c|c|c|c|c|c|c|c|c|}
\hline Problem & Algorithm & $\tilde{\eta}$ & $E R$ & $G D$ & Spacing & Max $_{\text {spread }}$ & $\begin{array}{l}\text { Sum of } \\
\text { weight }\end{array}$ & Rank \\
\hline \multirow{3}{*}{1} & MOGA & $15(2)$ & $0.5946(2)$ & $1.0340(1)$ & $1.2913(2)$ & $34.2251(2)$ & 9 & 2 \\
\hline & ACO & $12(1)$ & $0.6250(1)$ & $0.9694(2)$ & $1.1781(3)$ & $34.1030(1)$ & 8 & 3 \\
\hline & HGA & $24(3)$ & $0.2727(3)$ & $0.3565(3)$ & $2.2112(1)$ & $36.9763(3)$ & 13 & 1 \\
\hline \multirow{3}{*}{2} & MOGA & $22(2)$ & $0.4359(2)$ & $0.7180(2)$ & $1.0435(2)$ & $34.2097(3)$ & 11 & 2 \\
\hline & ACO & $11(1)$ & $0.6452(1)$ & $1.1590(1)$ & $1.4938(1)$ & $33.3108(2)$ & 6 & 3 \\
\hline & HGA & $35(3)$ & $0.1667(3)$ & $0.2681(3)$ & $0.8956(3)$ & $31.2778(1)$ & 13 & 1 \\
\hline \multirow{3}{*}{3} & MOGA & $12(2)$ & $0.7447(1)$ & $1.1370(2)$ & $1.0293(3)$ & $40.2682(2)$ & 10 & 2 \\
\hline & ACO & $10(1)$ & $0.6429(2)$ & $1.1764(1)$ & $1.8612(1)$ & $38.1782(1)$ & 6 & 3 \\
\hline & HGA & $40(3)$ & $0.1489(3)$ & $0.2517(3)$ & $1.4235(2)$ & $41.1657(3)$ & 14 & 1 \\
\hline \multirow{3}{*}{4} & MOGA & $29(2)$ & $0.3556(1)$ & $0.4950(2)$ & $1.3678(2)$ & $36.5902(1)$ & 8 & 3 \\
\hline & ACO & $26(1)$ & $0.2973(3)$ & $0.4366(3)$ & $1.7489(1)$ & $38.3385(2)$ & 10 & 2 \\
\hline & HGA & $35(3)$ & $0.3519(2)$ & $0.5074(1)$ & $1.1018(3)$ & $38.5984(3)$ & 12 & 1 \\
\hline \multirow{3}{*}{5} & MOGA & $11(2)$ & $0.5926(2)$ & $0.9460(2)$ & $1.2987(3)$ & $33.5636(2)$ & 11 & 2 \\
\hline & ACO & $10(1)$ & $0.6429(1)$ & $1.0924(1)$ & $1.3385(2)$ & $34.3586(3)$ & 8 & 3 \\
\hline & HGA & $22(3)$ & $0.2667(3)$ & $0.3276(3)$ & $1.6657(1)$ & $34.3586(3)$ & 13 & 1 \\
\hline \multirow{3}{*}{6} & MOGA & 13(1) & $0.7869(1)$ & 1.7381(1) & $1.6819(1)$ & $39.5870(1)$ & 5 & 3 \\
\hline & ACO & $25(3)$ & $0.5098(2)$ & $1.3454(2)$ & $1.6183(2)$ & $39.6918(2)$ & 10 & 2 \\
\hline & HGA & $40(2)$ & $0.4030(3)$ & $0.6576(3)$ & $1.4541(3)$ & $40.0436(3)$ & 15 & 1 \\
\hline \multirow{3}{*}{7} & MOGA & $16(2)$ & $0.6098(1)$ & $1.1300(1)$ & 1.6974(1) & $37.2650(1)$ & 6 & 3 \\
\hline & ACO & $16(2)$ & $0.5789(2)$ & $1.0099(2)$ & $1.5133(2)$ & $39.3564(3)$ & 11 & 2 \\
\hline & HGA & $41(3)$ & $0.3051(3)$ & $0.5166(3)$ & $1.1344(3)$ & $39.0404(2)$ & 14 & 1 \\
\hline \multirow{3}{*}{8} & MOGA & $17(2)$ & $0.7018(1)$ & $1.1665(1)$ & $1.3567(2)$ & 39.0331(3) & 9 & 2 \\
\hline & ACO & $16(1)$ & $0.5429(2)$ & $1.0410(2)$ & $1.8336(1)$ & $37.3966(1)$ & 7 & 3 \\
\hline & HGA & $40(3)$ & $0.3443(3)$ & $0.5396(3)$ & $0.9635(3)$ & $37.7713(2)$ & 14 & 1 \\
\hline \multirow{3}{*}{9} & MOGA & $17(2)$ & $0.6909(1)$ & 1.1311(1) & $1.1018(3)$ & $39.7311(2)$ & 9 & 2 \\
\hline & ACO & $14(1)$ & $0.5882(2)$ & $1.0600(2)$ & $1.6150(2)$ & $38.1256(1)$ & 8 & 3 \\
\hline & HGA & $36(3)$ & $0.4930(3)$ & $0.8795(3)$ & $1.7604(1)$ & $44.9119(3)$ & 13 & 1 \\
\hline \multirow{3}{*}{10} & MOGA & $16(1)$ & $0.6667(1)$ & $1.2655(1)$ & $1.5753(1)$ & $36.4029(1)$ & 5 & 3 \\
\hline & ACO & $25(2)$ & $0.5763(2)$ & $1.1164(2)$ & $1.3033(2)$ & $37.8426(3)$ & 11 & 2 \\
\hline & HGA & $30(3)$ & $0.5238(3)$ & $0.7406(3)$ & $1.0246(3)$ & $37.6970(2)$ & 14 & 1 \\
\hline
\end{tabular}




\begin{tabular}{|c|c|c|c|c|c|c|c|c|}
\hline Problem & Algorithm & $\tilde{\eta}$ & $E R$ & $G D$ & Spacing & Max $_{\text {spread }}$ & $\begin{array}{l}\text { Sum of } \\
\text { weight }\end{array}$ & Rank \\
\hline \multirow{3}{*}{11} & MOGA & 17(1) & $0.8496(1)$ & $1.9129(1)$ & $1.3149(3)$ & $45.1158(1)$ & 7 & 3 \\
\hline & ACO & $60(2)$ & $0.5000(2)$ & $0.9915(2)$ & $1.3560(2)$ & $46.4900(3)$ & 11 & 2 \\
\hline & $\mathrm{HGA}$ & $86(3)$ & $0.3723(3)$ & $0.7732(3)$ & $1.4197(1)$ & $45.4979(2)$ & 12 & 1 \\
\hline \multirow{3}{*}{12} & MOGA & $24(1)$ & $0.7073(1)$ & $1.7056(1)$ & $1.8150(1)$ & $48.4041(3)$ & 7 & 3 \\
\hline & ACO & $56(3)$ & $0.3253(3)$ & $0.6771(3)$ & $1.4082(3)$ & 46.8911(1) & 13 & 1 \\
\hline & HGA & $44(2)$ & $0.6271(2)$ & $1.3069(2)$ & $1.4886(2)$ & $47.8308(2)$ & 10 & 2 \\
\hline \multirow{3}{*}{13} & MOGA & $20(1)$ & $0.7701(1)$ & $1.6837(1)$ & $1.6846(2)$ & $48.4191(2)$ & 7 & 3 \\
\hline & ACO & $42(2)$ & $0.3731(2)$ & $0.7612(3)$ & $1.8370(1)$ & $47.0473(1)$ & 10 & 2 \\
\hline & HGA & $74(3)$ & $0.4351(3)$ & $0.8760(2)$ & $1.3466(3)$ & $49.9341(3)$ & 13 & 1 \\
\hline \multirow{3}{*}{14} & MOGA & $56(2)$ & $0.3778(3)$ & $0.8536(2)$ & $1.7669(1)$ & 53.7493(1) & 9 & 2 \\
\hline & ACO & $65(3)$ & $0.4348(2)$ & $0.8321(3)$ & $1.3163(2)$ & $54.6819(3)$ & 13 & 1 \\
\hline & HGA & $49(1)$ & $0.6797(1)$ & $1.4087(1)$ & $1.1580(3)$ & $53.8050(2)$ & 8 & 3 \\
\hline \multirow{3}{*}{15} & MOGA & $15(1)$ & $0.7857(1)$ & $2.0094(1)$ & $1.5541(3)$ & $50.1276(3)$ & 9 & 2 \\
\hline & ACO & $31(2)$ & $0.5441(2)$ & $1.1165(2)$ & $2.2496(1)$ & $49.3039(1)$ & 8 & 3 \\
\hline & HGA & $49(3)$ & $0.3194(3)$ & $0.7035(3)$ & $1.6625(2)$ & $49.6062(2)$ & 13 & 1 \\
\hline \multirow{3}{*}{16} & MOGA & $38(1)$ & $0.6696(1)$ & $1.6155(1)$ & $1.5986(2)$ & $55.3575(3)$ & 8 & 3 \\
\hline & ACO & $86(3)$ & $0.3723(3)$ & $0.7880(3)$ & $1.7949(1)$ & $55.2207(2)$ & 12 & 1 \\
\hline & HGA & $69(2)$ & $0.6124(2)$ & $1.4092(2)$ & $1.2798(3)$ & $54.1806(1)$ & 10 & 2 \\
\hline \multirow{3}{*}{17} & MOGA & $30(1)$ & $0.7391(1)$ & $1.9710(1)$ & $1.4983(3)$ & $55.7943(3)$ & 9 & 2 \\
\hline & ACO & $62(2)$ & $0.5000(3)$ & $1.2519(2)$ & $1.6083(1)$ & $55.1087(1)$ & 9 & 2 \\
\hline & HGA & $73(3)$ & $0.5494(2)$ & $1.2063(3)$ & $1.5393(2)$ & $55.7024(2)$ & 12 & 1 \\
\hline \multirow{3}{*}{18} & MOGA & $22(1)$ & $0.8182(1)$ & $1.9982(1)$ & $1.6087(2)$ & $57.2777(1)$ & 6 & 3 \\
\hline & ACO & $88(3)$ & $0.2000(3)$ & $0.5709(3)$ & $2.0579(1)$ & $59.0822(3)$ & 13 & 1 \\
\hline & HGA & $74(2)$ & $0.5747(2)$ & $1.4657(2)$ & $1.4820(3)$ & $57.3599(2)$ & 11 & 2 \\
\hline \multirow{3}{*}{19} & MOGA & $42(1)$ & $0.5922(1)$ & $1.6303(1)$ & $1.6454(3)$ & $57.3484(2)$ & 8 & 3 \\
\hline & ACO & $49(2)$ & $0.5664(2)$ & $1.4639(2)$ & $1.6960(2)$ & $57.1157(1)$ & 9 & 2 \\
\hline & HGA & $74(3)$ & $0.4559(3)$ & $1.0453(3)$ & $1.8645(1)$ & $58.5914(3)$ & 13 & 1 \\
\hline \multirow{3}{*}{20} & MOGA & $33(1)$ & $0.6972(1)$ & $1.5315(1)$ & $1.6453(2)$ & $61.8964(2)$ & 7 & 3 \\
\hline & ACO & $66(2)$ & $0.4000(3)$ & $0.9788(2)$ & $1.9603(1)$ & $61.3480(1)$ & 9 & 2 \\
\hline & HGA & $84(3)$ & $0.4650(2)$ & $0.8983(3)$ & $1.4834(3)$ & $63.2256(3)$ & 14 & 1 \\
\hline \multirow{3}{*}{21} & MOGA & $17(1)$ & $0.8411(1)$ & $2.2525(1)$ & $1.4918(2)$ & $56.7749(1)$ & 6 & 3 \\
\hline & ACO & $117(3)$ & $0.1761(3)$ & $0.3228(3)$ & $1.5736(1)$ & $58.4656(3)$ & 13 & 1 \\
\hline & HGA & $57(2)$ & $0.6780(2)$ & $1.7245(2)$ & $1.4484(3)$ & $58.2365(2)$ & 11 & 2 \\
\hline \multirow{3}{*}{22} & MOGA & $44(1)$ & $0.6944(1)$ & $1.6900(1)$ & $1.8500(2)$ & $62.3752(2)$ & 7 & 3 \\
\hline & ACO & $90(3)$ & $0.2857(3)$ & $0.8241(3)$ & $1.9158(1)$ & $65.5746(3)$ & 13 & 1 \\
\hline & HGA & $70(2)$ & $0.6410(2)$ & $1.5881(2)$ & $1.3136(3)$ & $62.1336(1)$ & 10 & 2 \\
\hline \multirow{3}{*}{23} & MOGA & $21(1)$ & $0.8397(1)$ & $2.4638(1)$ & $1.8718(2)$ & $63.7124(2)$ & 7 & 3 \\
\hline & ACO & $46(2)$ & $0.4458(3)$ & $1.1937(3)$ & $1.9034(1)$ & $64.4027(3)$ & 12 & 1 \\
\hline & $\mathrm{HGA}$ & $74(3)$ & $0.5912(2)$ & $1.9811(2)$ & $1.6302(3)$ & $63.6888(1)$ & 11 & 2 \\
\hline \multirow{3}{*}{24} & MOGA & $39(1)$ & $0.6286(2)$ & $1.6486(1)$ & $1.8588(2)$ & 64.5943(1) & 7 & 3 \\
\hline & ACO & 71(3) & $0.3238(3)$ & $0.8033(3)$ & $3.2717(1)$ & $67.5944(3)$ & 13 & 1 \\
\hline & HGA & $61(2)$ & $0.6494(1)$ & $1.6160(2)$ & $1.5992(3)$ & $65.5225(2)$ & 10 & 2 \\
\hline \multirow{3}{*}{25} & MOGA & $57(1)$ & $0.7077(2)$ & $2.1692(2)$ & $1.7039(2)$ & $72.1097(1)$ & 8 & 3 \\
\hline & ACO & $105(3)$ & $0.2606(3)$ & $0.5954(3)$ & $2.3302(1)$ & $73.1415(3)$ & 13 & 1 \\
\hline & HGA & $74(2)$ & $0.7218(1)$ & $2.3913(1)$ & $1.6459(3)$ & $72.5640(2)$ & 9 & 2 \\
\hline
\end{tabular}

Based on the result in Table 10, the HGA consistently show the best performance in all problems having low and low-medium graph difficulties (problem 1-10). Meanwhile, the MOGA show better performance compare with ACO algorithm for problem 1-3, but then then showed inconsistent performance for problem 4 to 10. In problem 4 to $10, \mathrm{ACO}$ algorithm starts 
to overcome the MOGA performance in some cases.

Meanwhile, for the problem with medium and medium-high graph difficulties (problem 11 - 20), the HGA and ACO algorithms alternately lead the algorithms in the first rank. However, when the graph difficulty is increased to high difficulty (problem 21 - 25), ACO has consistently shows better performance and then followed by HGA and MOGA. The relative performance of each algorithm is presented graphically in Figure 8.

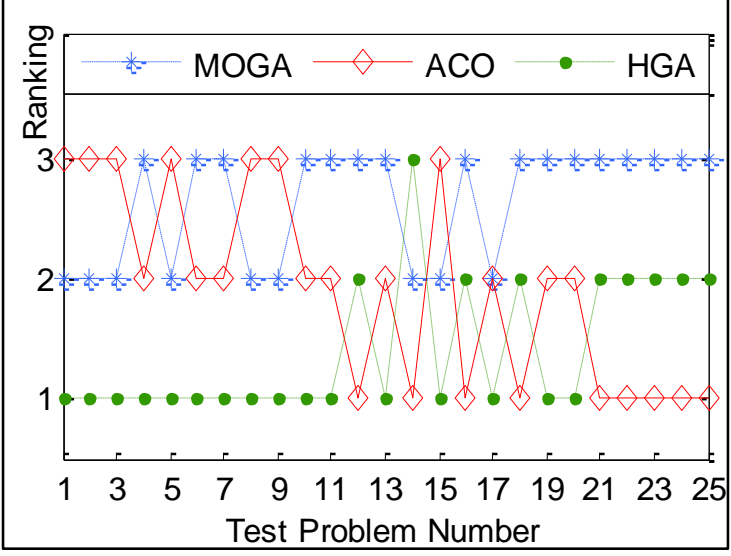

Figure 8: Algorithm's ranking for the range of test problems

\section{Conclusions}

In this work, a test problem generator (TPG) with tuneable complexity for ASP and ALB problems has been proposed. A set of experiments has been conducted to assess the TPG. Experimental results confirm that problem complexities can be controlled by tuneable input variables.

The results from Phase 1 experiments that test the effects of tuneable inputs confirm the ability of TPG to generate problem with varying complexity levels. The problem difficulties will increase when using larger number of tasks $(n)$, smaller Order Strength $(O S)$ value, larger Frequency Ratio $(F R)$ or smaller Time Variability ratio (TV). As presented in the results, the $n$ and $O S$ influence the assembly graph difficulties, while $F R$ and $T V$ influence the assembly data difficulties. The result of statistical test confirmed that there are significant differences of the problem difficulties when changing the value of $n$ and $T V$ variables. On the other hand, the significant difference of problem difficulties also can be achieved by selecting appropriate value for $O S$ and $F R$ as suggested in Table 2 .

Results of algorithm performance experiment in Phase 2 show that the Hybrid Genetic Algorithm (HGA) consistently performed well in optimising problem with low and medium difficulties. Meanwhile, the Ant Colony Optimisation (ACO) showed good performance in problems with high level of difficulty. Based on the performance of both algorithms, the HGA is recommended for integrated ASP and ALB problem with low and medium difficulties, while the ACO is for ASP and ALB problem at high difficulty. These findings confirm that the problems generated by the TPG offer sufficient range of problem variety to be used in algorithm testing. The generated problems were found to be useful to identify the strengths and weaknesses of the tested algorithms.

Although further experiments are needed to confirm these strengths and weaknesses, TPG has provided an important path by supplying a variety of ASP and ALB problems for systematic testing. Therefore, it can be concluded that the proposed test problem generator is able to generate combined ASP and ALB problems in a wide range of difficulties.

\section{References}

[1] Lu, C., Wong, Y. S. and Fuh, J. Y. H. (2006), "An enhanced assembly planning 
approach using a multi-objective genetic algorithm", Proceedings of the Institution of Mechanical Engineers, Part B: Journal of Engineering Manufacture, vol. 220, no. 2, pp. 255-272.

[2] Rashid, M. F. F., Hutabarat, W. and Tiwari, A. (2011), "A review on assembly sequence planning and assembly line balancing optimisation using soft computing approaches", International Journal of Advanced Manufacturing Technology, , pp. 1-15.

[3] Chen, R., Lu, K. and Yu, S. (2002), "A hybrid genetic algorithm approach on multi-objective of assembly planning problem", Engineering Applications of Artificial Intelligence, vol. 15, no. 5, pp. 447-457.

[4] Tseng, H. and Tang, C. (2006), "A sequential consideration for assembly sequence planning and assembly line balancing using the connector concept", International Journal of Production Research, vol. 44, no. 1, pp. 97-116.

[5] Marian, R. M., Luong, L. H. S. and Abhary, K. (2006), "A genetic algorithm for the optimisation of assembly sequences", Computers and Industrial Engineering, vol. 50, no. 4, pp. 503-527.

[6] Chica, M., Cordón, O., Damas, S. and Bautista, J. (2010), "Multiobjective constructive heuristics for the $1 / 3$ variant of the time and space assembly line balancing problem: ACO and random greedy search", Information Sciences, vol. 180, no. 18, pp. 3465-3487.

[7] Smith, S. S. -. (2004), "Using multiple genetic operators to reduce premature convergence in genetic assembly planning", Computers in Industry, vol. 54, no. 1 , pp. 35-49.

[8] Kilincci, 0. and Bayhan, G. M. (2006), "A Petri net approach for simple assembly line balancing problems", International Journal of Advanced
Manufacturing Technology, vol. 30, no. 11-12, pp. 1165-1173.

[9] De Fazio, T. L. and Whitney, D. E. (1987), "Simplified generation of all mechanical assembly sequences", IEEE Journal of Robotics and Automation, vol. RA-3, no. 6, pp. 640-658.

[10] Chen, S. and Liu, Y. (2001), "An adaptive genetic assembly-sequence planner", International Journal of Computer Integrated Manufacturing, vol. 14, no. 5, pp. 489-500.

[11] Smith, S. S. and Liu, Y. (2001), "The application of multi-level genetic algorithms in assembly planning", Journal of Industrial Technology, vol. 17, no. 4.

[12] Wang, J. F., Liu, J. H. and Zhong, Y. F. (2005), "A novel ant colony algorithm for assembly sequence planning", International Journal of Advanced Manufacturing Technology, vol. 25, no. 11-12, pp. 1137-1143.

[13] Scholl, A. (1993), "Data of Assembly Line Balancing Problem", Schriften zur Quantitativen Betriebswirtschaftslehre 16/1993, TU Darmstadt, vol. 16/1993.

[14] Rardin, R. L. and Uzsoy, R. (2001), "Experimental evaluation of heuristic optimization algorithms: A tutorial", Journal of Heuristics, vol. 7, no. 3, pp. 261-304.

[15] Bhattacharjee, T. K. and Sahu, S. (1990), "Complexity of single model assembly line balancing problems", Engineering Costs and Production Economics, vol. 18, no. 3, pp. 203-214.

[16] Otto, A., Otto, C. and Scholl, A. (2011), "SALBPGen - A systematic data generator for (simple) assembly line balancing", Jena Research Papers in Business and Economics, [Online], vol. 5, available at: pubdb.wiwi.unijena.de/pdf/wp-jbe201105.pdf. 
[17] Kilbridge, M. and Wester, L. (1961), "The Balance Delay Problem", Management Science, vol. 8, no. 1, pp. 6984.

[18] Panneton, F., L'Ecuyer, P. and Matsumoto, M. (2006), "Improved longperiod generators based on linear recurrences modulo 2", ACM Transactions on Mathematical Software, vol. 32, no. 1, pp. 1-16.

[19] Rashid, M. F. F., Tiwari, A. and Hutabarat, W. (2011), "An Integrated Representation Scheme for Assembly Sequence Planning and Assembly Line Balancing", Proceedings of the 9th International Conference of Manufacturing Research, ICMR 2011, 6-8 September 2011, Glasgow, UK, .

[20] Moon, C., Kim, J., Choi, G. and Seo, Y. (2002), "An efficient genetic algorithm for the traveling salesman problem with precedence constraints", European Journal of Operational Research, vol. 140, no. 3, pp. 606-617.

[21] Choi, Y., Lee, D. M. and Cho, Y. B. (2009), "An approach to multi-criteria assembly sequence planning using genetic algorithms", International Journal of Advanced Manufacturing Technology, vol. 42, no. 1-2, pp. 180-188.

[22] Bautista, J. and Pereira, J. (2007), "Ant algorithms for a time and space constrained assembly line balancing problem", European Journal of Operational Research, vol. 177, no. 3, pp. 2016-2032.

$\begin{array}{rrr}\text { [23] Deb K. } & \begin{array}{r}\text { (2001), } \\ \text { Optimization }\end{array} & \begin{array}{r}\text { Multi-Objective } \\ \text { Evolutionary }\end{array}\end{array}$
Algorithm, John Wiley \& Sons Inc., England.

[24] Yoosefelahi, A., Aminnayeri, M., Mosadegh, H. and Ardakani, H. D. "Type II robotic assembly line balancing problem: An evolution strategies algorithm for a multi-objective model", Journal of Manufacturing Systems, Article in Press.

[25] Mastor, A. (1970), "An Experimental Investigation and Comparative Evaluation of Production Line Balancing Techniques", Management Science, vol. 16 , no. 11, pp. 728-746.

[26] Johnson, R. (1981), "Assembly line balancing algorithms: computation comparisons", International Journal of Production Research, vol. 19, no. 3, pp. 277-287.

[27] Urban, T. L. and Chiang, W. (2006), "An optimal piecewise-linear program for the U-line balancing problem with stochastic task times", European Journal of Operational Research, vol. 168, no. 3, pp. 771-782.

[28] Coolidge, F. (2000), Statistics: A Gentle Introduction, SAGE Publication Ltd, London

[29] Bahalke U., Dolatkhahi K., Dehghani, Jahani, V Yazdanparast, and $\mathrm{H}$ Hajihosseini (2011), "Formulation and heuristic algorithm for flow time minimization in a simple assembly line", Proceedings of the Institution of Mechanical Engineers, Part B: Journal of Engineering Manufacture, Article in Press, DOI 0954405411422468. 